\title{
Requirements to Detect the Monoenergetic Photon Signature of Thermal Cold Dark Matter in PeV-Scale Split Supersymmetry
}

\author{
Brooks Thomas \\ Michigan Center for Theoretical Physics (MCTP) \\ Department of Physics, University of Michigan, Ann Arbor, MI 48109
}

March 23, 2005

\begin{abstract}
Split supersymmetry scenarios with PeV-scale scalar masses circumvent many of the restrictions on supersymmetry coming from experimental limits on $\mathrm{CP}$ violation, flavor-changing neutral currents, and the Higgs boson mass. We consider the situation where the LSP is either a Wino or Higgsino and the majority of its cold dark matter relic density is of thermal origin, in which case its mass is specified to be $2.3 \mathrm{TeV}$ for a Wino or $1.1 \mathrm{TeV}$ for a Higgsino. We examine the potential for indirect detection, at present and future $\gamma$-ray telescopes, of the monoenergetic photon lines that would result from the annihilation of these particles near the galactic center. We show how the possibility for detection depends on the precise form of the galactic dark-matter halo profile and examine what performance attributes a $\gamma$-ray detector would need in order to register a $5 \sigma$ discovery.
\end{abstract}

\section{Theory}

If we ignore philosophical arguments regarding 'naturalness', we can seriously consider a broader array of theories as being 'physical' in the sense that they could realistically describe our universe. Such a reconsideration is both theoretically plausible, in light of string/M-theory landscape considerations, and observationally motivated. Because of data pressures on supersymmetric theories arising from Higgs mass searches, limits on CP violation effects, etc., it would be advantageous if one could assign to all the scalars in the theory (with the exception of one light Higgs particle) masses at [1] or above [2, 3] the $\mathrm{PeV}$ scale while keeping the gauginos 
relatively light, with $\mathrm{TeV}$-scale masses, to constitute dark matter. Taking anomalymediation as the origin of gaugino masses, but not scalar masses [4, provides us with the desired mass hierarchy. If the chiral supermultiplet $S$ is charged under some symmetry, the scalar masses are generated via

$$
\mathcal{L} \supset \int d^{2} \theta d^{2} \bar{\theta} \frac{S^{\dagger} S}{M_{P}^{2}} \Phi_{i}^{\dagger} \Phi_{i} \longrightarrow \frac{F_{S}^{\dagger} F_{S}}{M_{P}^{2}} \phi_{i}^{*} \phi_{i},
$$

as usual, but the equation that would normally generate the gaugino masses

$$
\mathcal{L} \supset \int d^{2} \theta \frac{S}{M_{P}^{2}} W^{a} W_{a} \longrightarrow \frac{F_{S}}{M_{P}^{2}} \lambda^{a} \lambda_{a}
$$

is no longer gauge invariant. Instead, the leading contribution to the gaugino masses arises at the one-loop level [4] and takes the form

$$
M_{\lambda}=\frac{\beta_{g_{\lambda}}}{g_{\lambda}} \frac{\left\langle F_{S}^{\dagger} F_{S}\right\rangle}{M_{P}^{2}}
$$

where the index $\lambda$ labels the three Standard Model gauge groups. Explicit dependence of each gaugino mass on the beta function of the gauge group with which it is associated determines the ratio between them, which to lowest order is

$$
M_{3} \simeq 3 M_{1} \simeq 9 M_{2} .
$$

If indeed the gravitino mass $m_{3 / 2}^{2}=\left\langle F_{S}^{\dagger} F_{S}\right\rangle / M_{P}^{2}$ is at the PeV-scale all the scalar sparticles acquire $\mathrm{PeV}$-scale masses while the gaugino masses are kept light, around the $\mathrm{TeV}$ scale, in this construction, an example of what has come to be known as "split supersymmetry" 2, 3, 5. Since the gaugino mass hierarchy is determined by equation (4), the lightest supersymmetric particle must in general be some mixture of Wino and Higgsino (in more general split supersymmetry models, the LSP may also have a significant Bino fraction: this case is examined in [6]). The only parameter yet unspecified in this scenario that has any bearing on the identity of the LSP is the $\mu$ term. If $\mu>M_{2}$, the LSP is Wino-like; if $\mu<M_{2}$, it is Higgsino-like; and if the two are comparable, it will be a mixture of the two.

In this work, we examine the case in which the LSP is effectively either pure Wino or pure Higgsino, noting that if $m_{\mathrm{LSP}} \gg m_{Z}$, these two possibilities cover the vast majority of $\left(M_{2}, \mu\right)$ parameter space. If the Wino is the LSP, its thermal relic abundance [3] is given by

$$
\Omega_{\tilde{W}}^{\text {therm }} \simeq 0.02\left(\frac{M_{2}}{1 \mathrm{TeV}}\right)^{2}
$$


For a Higgsino LSP, the result is

$$
\Omega_{\tilde{H}}^{\text {therm }} \simeq 0.09\left(\frac{\mu}{1 \mathrm{TeV}}\right)^{2}
$$

We can use the current WMAP bound [7] on the abundance of cold dark matter in the universe

$$
\Omega_{\mathrm{CDM}} h^{2}=0.11 \pm 0.01 \text { (WMAP } 68 \% \text { C.L.) }
$$

to find the mass the LSP would require in order to have an interesting relic density. In this paper, we will make the simplifying assumptions that this particle makes up all the dark matter in the universe and that its relic density is generated thermally. the result, in each case, is

$$
\begin{aligned}
& M_{\tilde{W}} \simeq 2.3 \pm 0.2 \mathrm{TeV} \\
& M_{\tilde{H}} \simeq 1.1 \pm 0.1 \mathrm{TeV} .
\end{aligned}
$$

If we allow for the possibility of other dark matter constituents, or for nonthermal LSP generation, these equations become an upper bound. In the purely thermal case, it should be noted that in order to make up even $20 \%$ of the dark matter in the universe, $M_{\tilde{W}}$ would still need to exceed $1 \mathrm{TeV}\left(M_{\tilde{H}}\right.$ would be around $\left.500 \mathrm{GeV}\right)$ and that none of the particle properties relevant to the indirect detection methods discussed below (e.g. annihilation cross-section into $\gamma$-rays) varies significantly over this mass range. If there are nonthermal contributions to the LSP relic density, however, any value of $M_{\text {LSP }}$ below the bound given by equations (8) and (9) is permitted.

Since theory has stipulated a TeV-scale Wino or Higgsino as our dark matter candidate, it is reasonable to ask whether any signature of such a particle could be detected experimentally. Direct detection of such a massive Wino or Higgsino LSP is effectively ruled out for all but the most unnaturally peaked halo models ${ }^{1}$ and the positron signal resulting from their annihilation would be too small to detect. The only truly promising detection method is to search for high-energy photons produced monoenergetically by Wino annihilation in the galactic halo. These photons would have energies of

$$
E_{\gamma}=m_{\chi} \quad \text { and } \quad E_{\gamma}=m_{\chi}\left(1-\frac{m_{Z}^{2}}{4 m_{\chi}^{2}}\right),
$$

\footnotetext{
${ }^{1}$ A thorough exposition of LSP dark matter constraints from various planned and operational experiments, especially in mixed Wino-Higgsino and Bino-Wino scenarios, can be found in 8 .
} 
(corresponding to $\chi \chi \rightarrow \gamma \gamma$ and $\chi \chi \rightarrow \gamma Z$ processes respectively). Presumably, as the concentration of dark matter is greatest in the center of the galaxy, one could aim a sufficiently powerful $\gamma$-ray telescope at the galactic center and see a signal at around $2.3 \mathrm{TeV}$. Several recent papers [8, 9, 10, have discussed the feasibility of detecting such a signal, for lower-scale AMSB scenarios at currently functioning or currently planned detectors. Our approach will be rather to assume the specific, $\mathrm{PeV}$-scale theory outlined above and assess what performance attributes would render a detector capable of observing the $\gamma$-ray signature of such particles.

The cross-sections for the annihilation for a pair of Winos or Higgsinos into a pair of photons and into a photon and a Z in anomaly-mediated SUSY-breaking scenarios have been examined by several authors [11. In both the Wino and Higgsino cases, each cross-section tends toward an asymptotic value as $m_{\text {LSP }}$ increases. For $m_{\text {LSP }}$ greater than a few hundred $\mathrm{GeV}, \sigma v(\gamma \gamma)$ and $\sigma v(Z \gamma)$ may effectively be replaced with their asymptotic values, which for a Wino LSP are:

$$
\begin{aligned}
\sigma_{\tilde{W}} v(\gamma \gamma) & \simeq 4.0 \times 10^{-27} \mathrm{~cm}^{-3} \mathrm{~s}^{-1} \\
\sigma_{\tilde{W}} v(Z \gamma) & \simeq 9.0 \times 10^{-27} \mathrm{~cm}^{-3} \mathrm{~s}^{-1}
\end{aligned}
$$

and for a Higgsino LSP, are

$$
\begin{aligned}
\sigma_{\tilde{H}} v(\gamma \gamma) & \simeq 9.0 \times 10^{-29} \mathrm{~cm}^{-3} \mathrm{~s}^{-1} \\
\sigma_{\tilde{H}} v(Z \gamma) & \simeq 2.0 \times 10^{-29} \mathrm{~cm}^{-3} \mathrm{~s}^{-1} .
\end{aligned}
$$

To be able to resolve the two lines would be an excellent test of the theory, but to do so for a $2.3 \mathrm{TeV}$ Wino would require an energy resolution $\Delta E / E$ of better than $3.5 \%$. Although future satellite facilities comparable to GLAST may have this kind of energy resolution, these telescopes are unlikely to discover dark matter from $\mathrm{PeV}$ scale split supersymmetry for reasons we shall soon make clear. For ground-based detectors, which have far coarser energy resolutions (on the order of 10-20\%), the two signals will be indistinguishable and add together to form a single 'line'.

\section{Halo Models and Dark Matter Distribution}

While the particle properties (mass, cross-section, etc.) of PeV-scale AMSB dark matter are more or less specified by the theory we have chosen, the distribution of that dark matter in the galaxy has yet to be specified. The shape of this distribution is important in determining the requirements for indirect detection of a $2.3 \mathrm{TeV}$ Wino, but is also not well known. Thus, rather than choosing any particular model, 
we focus on a representative set of halo profiles. These profiles are derived from numerical simulations [12, 13, and most take the form

$$
\rho(r)=\frac{\rho_{0}}{(r / R)^{\gamma}\left(1+(r / R)^{\alpha}\right)^{(\beta-\gamma) / \alpha}},
$$

where the three power-law indices $\alpha, \beta$, and $\gamma$, along with the characteristic radius $\mathrm{R}$, define a given model. The models we examine here include the Moore et al. profile (a relatively cuspy model), a pair of isothermal models, one with a smooth density distribution, the other with some clumping of the dark matter, and the widely used Navarro-Frenk-White profile. The choice of $\alpha, \beta$, and $\gamma$ which defines each model is given in table 1. In addition to these, we include in our analysis the halo profile proposed by Burkert et al. 14, for which the CDM density is modeled not by equation (15), but by

$$
\rho(r)=\frac{\rho_{0} r_{0}^{3}}{\left(r-r_{0}\right)\left(r^{2}-r_{0}^{2}\right)},
$$

where $r_{0}$ is a fiducial distance parameter. It has also been suggested that the presence of a massive black hole at the center of the galaxy could significantly alter the halo profile through the addition of a density spike at the galactic center [15]. However, as there is some debate over the precise effect the black hole would have, we will not consider this situation here.

For a given distribution of dark matter $\rho(r, \psi)$, the observed integral flux of $\gamma$-rays (usually expressed in $\mathrm{cm}^{-2} s^{-1}$ ) from LSP annihilations around the galactic center is

$$
\Phi=(\sigma v) n_{\gamma} \frac{1}{4 \pi m_{\chi}} \int_{L} \rho^{2}(\psi, s) d s,
$$

where $\rho$, the CDM mass density, depends on both the line-of-sight distance element $s$, the number $n_{\gamma}$ of photons produced per decay, and the angle $\psi$ away from the galactic center, and the integral is evaluated along the line of sight. It is common to abstract the density integral, which depends on the halo profile but is independent of the particle physics, by defining the quantity

$$
J(\psi) \equiv \frac{1}{8.5 \mathrm{kpc}}\left(\frac{1}{0.3 \mathrm{GeV}}\right)^{2} \int_{L} \rho^{2}(\psi, s) d s .
$$

For a detector with angular acceptance $\Delta \Omega$, the relevant quantity is not $J(0)$, but rather $\langle J(\psi)\rangle_{\Delta \Omega}$, the average of $J(\psi)$ over the solid angle given by $\Delta \Omega$. This quantity depends only on the value of $\Delta \Omega$ and the choice of halo profile. In figure 1, we show the relationship between $\langle J(\psi)\rangle_{\Delta \Omega}$ and $\Delta \Omega$ for some of the most commonly used halo models. 


\begin{tabular}{|l|cccc|}
\hline & $\alpha$ & $\beta$ & $\gamma$ & $R$ \\
\hline Isothermal profiles & 2.0 & 2.0 & 0 & 3.5 \\
NFW & 1.0 & 3.0 & 1.0 & 20.0 \\
Moore et al. & 1.5 & 3.5 & 1.5 & 28.0 \\
\hline
\end{tabular}

Table 1: The defining parameters $\alpha, \beta, \gamma$ and $R$ (see equation 15) for the halo models we examine. $R$ is given in kpc.

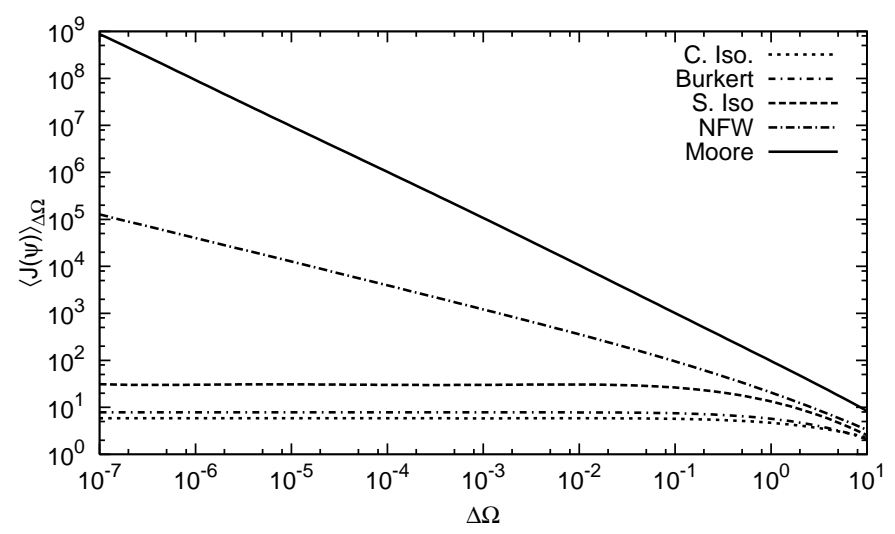

Figure 1: $\langle J(\psi)\rangle_{\Delta \Omega}$ (the line-of-sight integral through the halo density squared, averaged over the angular acceptance $\Delta \Omega$ ) vs. $\Delta \Omega$ for several halo profiles: smooth isothermal, clumpy isothermal, Burkert, NFW, and Moore et al.

\section{Detection and Instrumental Limitations}

We now turn to a discussion of the properties of $\gamma$-ray detectors and the physical limitations to which they are subject. The relevant quantities, in terms of telescope performance, are effective area, energy resolution, angular resolution, and field of view. Significant improvements in energy resolution could effectively cut the background in the energy range of interest by an order of magnitude (see figures 2 and 3), while better angular resolution can improve the signal-to-background ratio, depending on the halo profile. For a cuspy profile, such as the Moore et al. or NFW profile, $\langle J(\psi)\rangle_{\Delta \Omega}$ increases rapidly with decreasing $\Delta \Omega$ and minimizing the angular acceptance enhances the visibility of the signal. For a less cuspy profile, the signal-to-background ratio decreases as $\Delta \Omega$ becomes smaller and the observation strategy would then be to relax the angular acceptance as much as possible within the detector's field of view. 


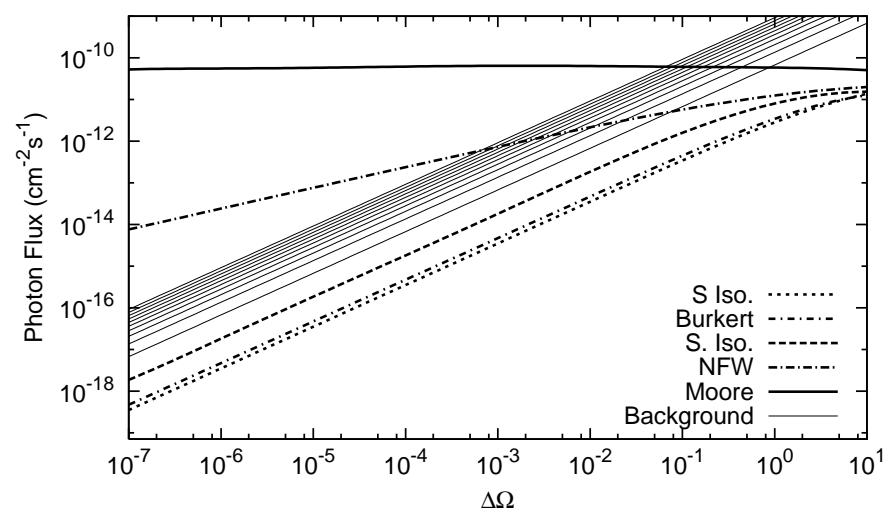

Figure 2: The expected flux from the annihilation of $2.3 \mathrm{TeV}$ Winos, as a function of $\Delta \Omega$, that would be detected by a satellite detector aimed at the galactic center. Also included is the anticipated background flux at such a detector for different values of detector energy resolution, ranging from $\Delta E / E=50 \%$ (top line) to $\Delta E / E=5 \%$ (bottom line). It should be noted that the spread in the signal is smaller than the width of the energy bin. From this, it is apparent that for the NFW and Moore et al. profiles, the prospects for detection increase with better angular resolution (decreasing $\Delta \Omega$ ). For a $1.1 \mathrm{TeV}$ Higgsino LSP, the resulting curves are similar, but the signal is two orders of magnitude lower, and the background flux is increased by a factor of $\sim 10$.

Satellite detectors, such as the soon-to-be-launched pair-production telescope GLAST, can have excellent angular resolution and energy resolution. The field of view for GLAST will be on the order of a steradian, its angular resolution in the $\mathrm{TeV}$ range will be $\sim 0.1^{\circ}$, and its energy resolution $\Delta E / E$ on the order of $4 \%$. Despite all this, all space-based telescopes are limited by collection area constraints ( $A_{\text {eff }}$ tends to be on the order of $10^{4} \mathrm{~cm}^{2}$ ), which will make it difficult for any space telescope to detect a large enough number photons in the $\mathrm{TeV}$ range to register a $5 \sigma$ discovery.

In contrast, atmospheric Cherenkhov detectors (ACTs) such as the HESS array, which operate by observing showers of Cherenkhov light that result when highenergy $\gamma$-rays enter the Earth's upper atmosphere, can have far larger effective areas. The area of the Cherenkhov light pool on the ground is $\sim 5 \times 10^{8} \mathrm{~cm}^{2}$, which defines the scale of the detector's collection area, which is comparatively enormous. However, uncertainties in reconstructing the energy of the primary photon from the properties of the radiation shower place limits on the energy resolution (a single 




Figure 3: The expected flux from the annihilation of $2.3 \mathrm{TeV}$ Winos, as a function of $\Delta \Omega$, that would be detected by a Cherenkhov detector aimed at the galactic center. Also included is the anticipated background flux at such a detector for different values of detector energy resolution, ranging from $\Delta E / E=50 \%$ (top line) to $\Delta E / E=5 \%$ (bottom line). It should be noted that the spread in the signal is smaller than the width of the energy bin. From this, it is apparent that for the NFW and Moore et al. profiles, the prospects for detection increase with better angular resolution (decreasing $\Delta \Omega$ ). For a $1.1 \mathrm{TeV}$ Higgsino LSP, the resulting curves are similar, but the signal is two orders of magnitude lower, and the background flux is increased by a factor of $\sim 10$.

imaging detector can achieve $\Delta E / E \simeq 30-40 \%$; an array of parallel detectors, $10-15 \%)$. Still, because of their large collection area, ACTs are probably the best bet for the detection of Wino or Higgsino dark matter from PeV-scale split supersymmetry.

In addition to the performance attributes discussed above, space-based and ground-based telescopes also 'see' different backgrounds. For a satellite detector such as GLAST, the background is the actual diffuse gamma-ray background, which for energies in the $\mathrm{TeV}$ range is less well-known. The best that can currently be done is to make the assumption one can extrapolate the power law spectrum from EGRET data (good up to $~ 100 \mathrm{GeV}$ ) to higher energies [16, 17, 18, which yields a power-law of the form

$$
\frac{d n_{\mathrm{BG}}}{d \Omega d E}=N_{0}\left(\frac{E}{1 \mathrm{GeV}}\right)^{-\alpha} \mathrm{cm}^{-2} \mathrm{~s}^{-1} \mathrm{GeV}^{-1} \mathrm{sr}^{-1}
$$

with a numerical prefactor $N_{0}$ on the order of $10^{-6} \mathrm{~cm}^{2}$ and an exponent $\alpha$ some- 


\begin{tabular}{|l|cccc|}
\hline & $A_{\text {eff }}$ & $\Delta E / E$ & $\Delta \Omega_{\min }$ & $\epsilon_{\text {had }}$ \\
\hline WHIPPLE (Arizona) & $3.5 \times 10^{8}$ & $30 \%$ & $1.88 \times 10^{-5}$ & 1.0 \\
GRANITE II (Arizona) & $5 \times 10^{8}$ & $20 \%$ & $9.56 \times 10^{-6}$ & 1.0 \\
HESS (Namibia) & $7 \times 10^{8}$ & $15 \%$ & $9.56 \times 10^{-6}$ & 0.25 \\
VERITAS (Arizona) & $1 \times 10^{9}$ & $15 \%$ & $3.83 \times 10^{-7}$ & 0.25 \\
EGRET (Satellite) & $1 \times 10^{4}$ & $15 \%$ & $3.22 \times 10^{-2}$ & - \\
GLAST (Satellite) & $1.5 \times 10^{4}$ & $4 \%$ & $9.56 \times 10^{-6}$ & - \\
\hline Generic ACT & $1.5 \times 10^{9}$ & $10 \%$ & $1.00 \times 10^{7}$ & 0.25 \\
Generic PPT & $2 \times 10^{4}$ & $1 \%$ & $1.00 \times 10^{-7}$ & - \\
\hline
\end{tabular}

Table 2: The performance parameters for current and planned $\gamma$-ray telescopes, including both ACTs (WHIPPLE, GRANITE II, HESS, and VERITAS) and space telescopes (EGRET and GLAST). Also included are the parameters used for the generic atmospheric Cerenkhov telescope (ACT) and space-based pair production telescope (PPT) we have used in our analysis.

where between 2.0 and 2.5. Of course GLAST, when it turns on, will also provide a great deal of information on the diffuse $\gamma$-ray background at high energies, which will dramatically reduce the uncertainty in $\alpha$ and $N_{0}$.

The background for atmospheric Cherenkhov detectors consists mainly of cascade events triggered by cosmic-ray protons, electrons, etc., which dominate (by an order or two of magnitude) over the diffuse gamma-ray background. Showers initiated by leptons (predominately electrons) are indistinguishable from gamma-ray cascades, whereas hadronic showers can be differentiated to a degree due to the cascade's shape and to the time spread of the light pulse. While these backgrounds are higher than those seen by satellite detectors, their spectra are reliably known up to $5 \mathrm{TeV}$. The power-law behavior 20] for each is given below:

$$
\begin{gathered}
\frac{d N_{\mathrm{had}}}{d E d \Omega}=1.0 \cdot 10^{-2} \epsilon_{\mathrm{had}}\left(\frac{E_{0}}{1 \mathrm{GeV}}\right)^{-2.7} \mathrm{~cm}^{-2} \mathrm{~s}^{-1} \mathrm{GeV}^{-1} \mathrm{sr}^{-1} \\
\frac{d N_{\mathrm{e}^{-}}}{d E d \Omega}=6.9 \cdot 10^{-2}\left(\frac{E_{0}}{1 \mathrm{GeV}}\right)^{-3.3} \mathrm{~cm}^{-2} \mathrm{~s}^{-1} \mathrm{GeV}^{-1} \mathrm{sr}^{-1},
\end{gathered}
$$

where we have replaced $N_{0}$ and the power-law index $\alpha$ with their explicit numerical values. The factor of $\epsilon_{\text {had }}$ has been included to account for improved hadronic rejection techniques (the default value of $\epsilon_{\text {had }}=1$ corresponds to the Whipple telescope: instruments such as HESS and VERITAS have already improved on this by a factor of four). 
In figures 2 and 3, we show the expected gamma-ray flux from the $2.3 \mathrm{TeV}$ line as a function of angular acceptance, along with the expected background flux at a generic ACT and satellite detector whose performance attributes are slightly better than those of any currently planned facility (see table 2 for performance specifics) for a variety of different energy resolutions ranging from $5 \%$ to $50 \%$. It is apparent that the integral background flux seen by a satellite detector will generally be at least two orders of magnitude below that seen by an ACT, regardless of the precise power-law form of the diffuse $\gamma$-ray background.

In order for the signal registered at any detector to be interpreted as a discovery, two conditions must be met: first, the significance level (the ratio of $N_{S}$, the total number of signal photons registered, to $\sqrt{N_{\mathrm{BG}}}$, where $N_{\mathrm{BG}}$ is the total number of background photons registered) must exceed $5 \sigma$; second, the total number of detected photons must exceed 25, the threshold below which Poisson statistics give an equivalent confidence limit ${ }^{2}$. These requirements, when written explicitly in terms of $A_{\text {eff }}, \Delta E / E, \Delta \Omega$, and observation time, are

$$
\begin{gathered}
(.68)^{2}\left(\frac{\Phi(\Delta \Omega) \sqrt{A_{\text {eff }}}}{\sqrt{\Phi_{\mathrm{BG}}\left(\Delta \Omega, \Delta E / E, \epsilon_{\text {had }}\right)}}\right) \geq 5 \\
\Phi(\Delta \Omega) A_{\text {eff }} \geq 25
\end{gathered}
$$

Because the total number of collected photons is directly proportional to the collecting area, the second criterion implies that an ACT, with a characteristic $A_{\text {eff }}$ on the order of $10^{8}-10^{9} \mathrm{~cm}^{2}$, will be a more useful for detecting a PeV-scale dark matter signal than a satellite detector with an $A_{\text {eff }}$ of $\sim 10^{4} \mathrm{~cm}^{2}$, despite the smaller background seen by the latter. In figures 4 and 5 , we plot the the total number of signal photons recorded by our generic satellite detector and Cherenkhov array for a range of $\Delta \Omega$ and an exposure time of $10^{7} \mathrm{~s}$, in both the Wino and Higgsino LSP cases. Even with the liberal assumptions of a Wino-like LSP and a galactic halo density described by the Moore et al. profile, our telescope (and hence also GLAST) would not register a sufficient number of photons to signal a discovery.

The situation is far more hopeful for Cherenkhov detectors. Since the field of view an ACT can attain is on the order of $10^{-3} \mathrm{~cm}^{2}$, detection would still be out of reach if the dark matter distribution was less sharply cusped (such as in the isothermal and Burkert profiles), but would be possible for a more concentrated dark matter halo. It is an interesting coincidence that the NFW profile nearly demarcates the line between detection and non-detection for presently operational facilities: if

\footnotetext{
${ }^{2}$ While the likelihood of random statistical fluctuations at the $5 \sigma$ level increases with improved energy resolution, these can be differentiated from a true signal by requiring the signal to be consistent over multiple trials.
} 

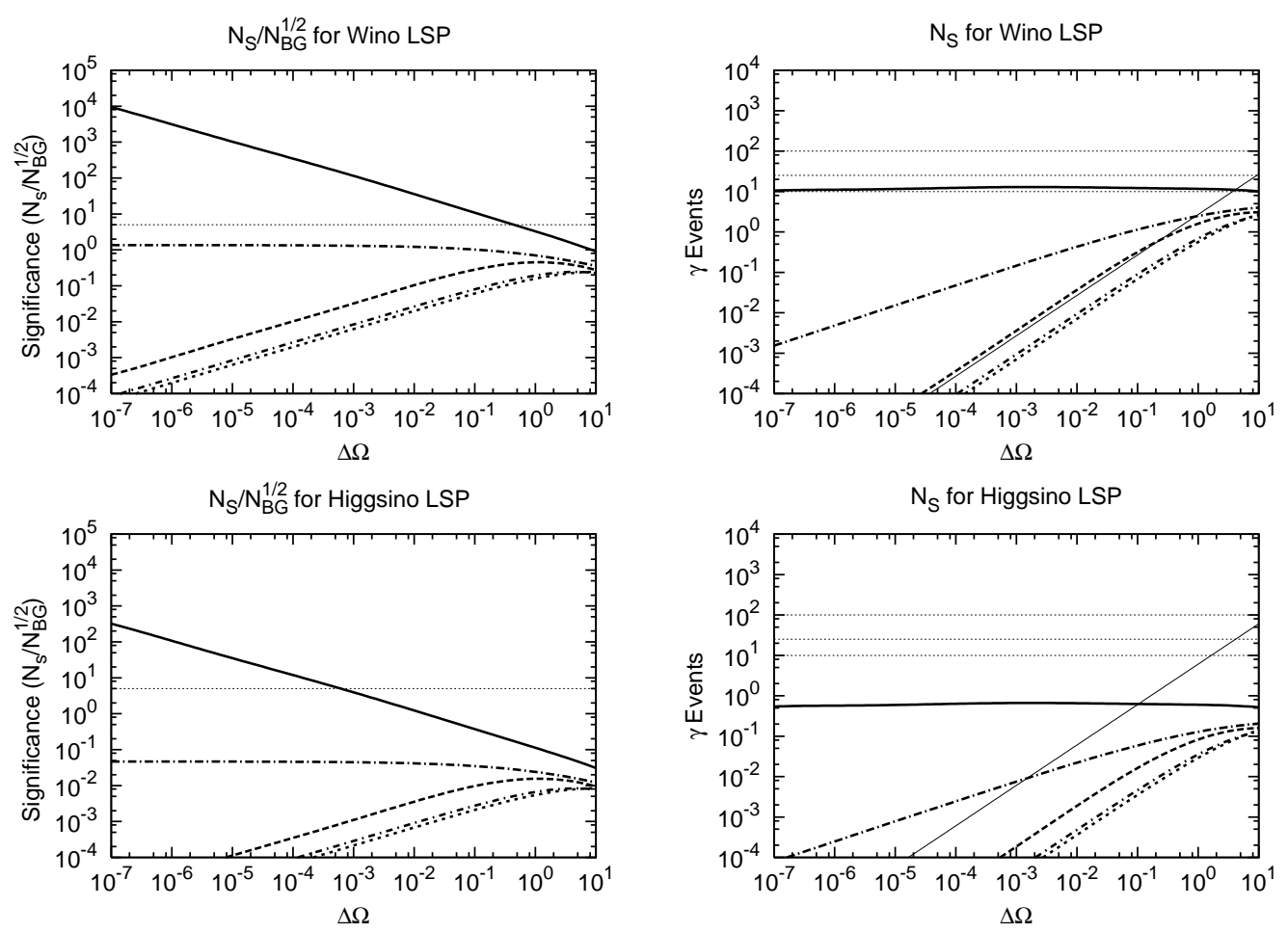

Figure 4: The ratio of $N_{\text {signal }} / \sqrt{N_{\text {background }}}$ (left panels) and total number of photons (right panels) collected by a generic space telescope with an effective area of $2 \times$ $10^{4} \mathrm{~cm}^{2}$ and an energy resolution of $1 \%$, over a range of $\Delta \Omega$, after $10^{7} \mathrm{~s}$ (about $1 / 3$ of an active year) of viewing time, and for both $2.3 \mathrm{TeV}$ Wino (top panels) and 1.1 TeV Higgsino LSP (bottom panels). See figure 3 caption for the halo model key. The threshold for $5 \sigma$ discovery has been included for reference in the significance graphs, and contours corresponding to 10, 25, and 100 events have been included in the event count graphs. It can be seen here that for such a space telescope, no halo profile is capable of producing the 25 events necessary for detection, and that only for the Moore et al. profile is the significance criterion even achieved.

the actual dark matter distribution is cuspier than that given by the NFW profile, the $\gamma$-ray signature of Wino dark matter in PeV-scale split supersymmetry should be detectable at the next generation of ACTs; if the actual profile is much less sharply peaked (e.g. if it resembles the smooth isothermal case), it is unlikely that such a signal would ever be detectable at an ACT. It should also be noted that a 1.1 TeV Higgsino LSP would be more difficult to detect than a $2.3 \mathrm{TeV}$ Wino (only 

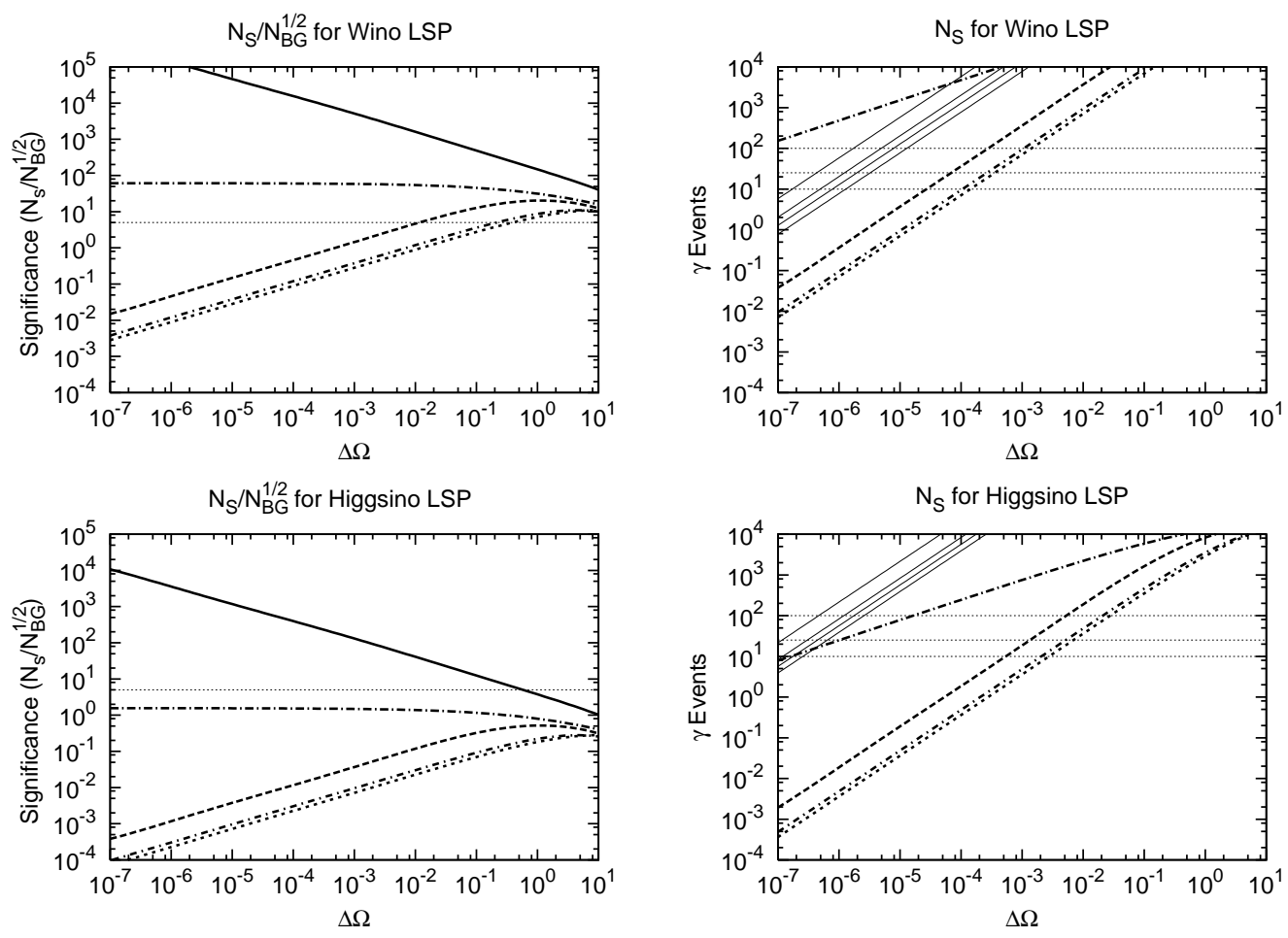

Figure 5: The ratio of $N_{\text {signal }} / \sqrt{N_{\text {background }}}$ (left panels) and total number of photons (right panels) collected by a generic Cherenkhov array with an effective area of $1.5 \times 10^{9} \mathrm{~cm}^{2}$ and an energy resolution of $10 \%$, over a range of $\Delta \Omega$, after $10^{7} \mathrm{~s}$ (about $1 / 3$ of an active year) of viewing time, and for both $2.3 \mathrm{TeV}$ Wino (top panels) and 1.1 TeV Higgsino LSP (bottom panels). See figure 3 caption for the halo model key. The threshold for $5 \sigma$ discovery has been included for reference in the significance graphs, and contours corresponding to 10, 25, and 100 events have been included in the event count graphs. It can be seen here that there are real prospects for detection with such a Cherenkhov detector, provided that the galactic CDM halo density resembles the NFW or Moore et al. profiles.

for the Moore et al. profile is the $5 \sigma$ requirement from equation (22) satisfied), both because the annihilation cross-section into $\gamma$-rays is smaller and because the background is around a factor of ten higher ${ }^{3}$ at $1.1 \mathrm{TeV}$ than at $2.3 \mathrm{TeV}$.

\footnotetext{
${ }^{3}$ This follows simply because the background photon spectrum obeys a power-law. Although the power-law index $\alpha$ is slightly different for ACTs and satellite detectors (see equation (20) and discussion below (19), the results for both are comparable.
} 


\section{$4 \quad$ Future Detectors}


Figure 6: Detection boundary contours in $A_{\text {eff- }} \Delta \Omega$ parameter space for the $\gamma$-ray signature of a $2.3 \mathrm{TeV}$ Wino, based on the $5 \sigma$ significance requirement from equation 22 for VERITAS (top left panel), with $A_{\mathrm{eff}}=1 \times 10^{9} \mathrm{~cm}^{2}$ and $\Delta E / E$, and GLAST (bottom left panel), with $A_{\text {eff }}=1.5 \times 10^{4} \mathrm{~cm}^{2}$; and based on the $N_{S} \geq 25$ event count requirement from equation 23 (right panel), for a variety of halo profiles. Bars showing the range of angular acceptances that can be chosen at VERITAS and GLAST have also been included. In order to register a discovery at either of these facilities for a given halo model, both the $5 \sigma$ and $N_{s} \geq 25$ contours for that model must lie below the bar corresponding to that facility.

An interesting question to ask, especially if current facilities would be unable to register a discovery, is what attributes would a detector need in order to conclusively discover Wino or Higgsino dark matter in a PeV-scale split supersymmetry scenario. Since collection area and angular acceptance (which may be adjusted as desired over a window ranging from a detector's angular resolution (in steradians) to its field of view) have the most significant effect on the detection capabilities of any given 
instrument, we will focus on the effect of improvements in $A_{\text {eff }}$ and $\Delta \Omega$. In figure 6 we show the contours defined by equations (22) and (23) in $A_{\text {eff }}-\Delta \Omega$ space for $10^{7} \mathrm{~s}$ of viewing time, where the $\Delta E / E$ values used in computing the significance limits are those for the GLAST telescope and the VERITAS array (see table 2), along with the attributes of GLAST and VERITAS themselves, which appear as bars due to our freedom to adjust $\Delta \Omega$. The criterion for discovery at either of these facilities is that for a given halo model, both the $5 \sigma$ and $N_{s} \geq 25$ contours for that model lie below the corresponding bar. From this figure, it is evident that GLAST, primarily due to its small effective area, would be unable to detect CDM from PeV-scale split supersymmetry at all. Detection would be possible at VERITAS, provided that the dark matter distribution isn't too much flatter than the NFW profile. If the halo resembles the Burkert or isothermal profiles, however, neither facility (and no telescope present or currently planned) would be able to register a $5 \sigma$ discovery.

Because of their inherently large collection areas, Cherenkhov detectors are currently the best bet for the discovery of dark matter from PeV-scale split supersymmetry. The collection area of an ACT may be increased by the addition of more telescopes in the array, but the increment supplied by each individual telescope is merely additive, meaning it would not be feasible to raise $A_{\text {eff }}$ by two orders of magnitude over that of VERITAS. It would, however, be possible to engineer an ACT with a larger field of view: the fields of view for present and planned facilities are kept on the order of $10^{-3}$ sr primarily as a mechanism to deal with the effects of secondary electron scattering on the detector's low-energy response which will not affect observations in the $\mathrm{TeV}$ range. Operating at a large $\Delta \Omega$, such an instrument would be able to register a discovery for a range of more gently sloping halo profiles.

Since ACTs are intrinsically limited in energy resolution to $\Delta \Omega \sim 10 \%$ by uncertainties in reconstructing the energy of the initial photon from its cascade products [19], future facilities would still be unable to see the telltale indication that an observed $\gamma$-ray signal is the result of dark matter annihilation: the resolution of the $Z \gamma$ and $\gamma \gamma$ lines. Satellite detectors are already approaching this level of energy resolution, and so it is of particular interest whether a space-based facility could ever be built that would be able to detect them ${ }^{4}$. The difficulty, of course, is that even count considerations would mandate that the detection array be at least $100 \mathrm{~m}$ on a side, and that keeping such an object operational for $10^{7} \mathrm{~s}$ (considering potential disasters relating to solar wind, space debris, etc. that such an object would face), let alone launching it into into space, amount to a nearly insurmountable problem. It might be possible someday to construct such a detection array on the far side of

\footnotetext{
${ }^{4}$ Another advantage satellite detectors have over their ground-based counterparts is that their large fields of view could in principle allow for detection in even the most conservative of halo models.
} 
the moon, where the weight and area of the detector would not be an impediment, but unless a project of this sort were undertaken, it is unlikely that we would ever see the conclusive double-line signal of dark matter in PeV-scale split supersymmetry.

\section{Acknowledgements}

I would like to thank Tim McKay, Ting Wang, and James Wells for useful discussions.

\section{References}

[1] J. D. Wells, hep-ph/0306127; J. D. Wells, hep-ph/0411041.

[2] N. Arkani-Hamed and S. Dimopoulos, hep-th/0405159; N. Arkani-Hamed, S. Dimopoulos, G. F. Giudice and A. Romanino, hep-ph/0409232.

[3] G. F. Giudice and A. Romanino, Nucl. Phys. B 699, 65 (2004) [Erratum-ibid. B 706, 65 (2005)] hep-ph/0406088.

[4] L. Randall and R. Sundrum, Nucl. Phys. B 557, 79 (1999) hep-th/9810155; G. F. Giudice, M. A. Luty, H. Murayama and R. Rattazzi, JHEP 9812, 027 (1998) hep-ph/9810442.

[5] A. Arvanitaki, C. Davis, P. W. Graham and J. G. Wacker, Phys. Rev. D 70, 117703 (2004) hep-ph/0406034.

[6] A. Pierce, Phys. Rev. D 70, 075006 (2004) hep-ph/0406144.

[7] D. N. Spergel et al. [WMAP Collaboration], Astrophys. J. Suppl. 148, 175 (2003) astro-ph/0302209.

[8] A. Masiero, S. Profumo and P. Ullio, hep-ph/0412058.

[9] P. Ullio, JHEP 0106, 053 (2001) hep-ph/0105052.

[10] A. Arvanitaki and P. W. Graham, hep-ph/0411376.

[11] L. Bergstrom and P. Ullio, Nucl. Phys. B 504, 27 (1997) hep-ph/9706232;

Z. Bern, P. Gondolo and M. Perelstein, Phys. Lett. B 411, 86 (1997) hep-ph/9706538.

[12] B. Moore, T. Quinn, F. Governato, J. Stadel and G. Lake, Mon. Not. Roy. Astron. Soc. 310, 1147 (1999) astro-ph/9903164. 
[13] J. F. Navarro, C. S. Frenk and S. D. M. White, Astrophys. J. 462, 563 (1996) astro-ph/9508025.

[14] A. Burkert, IAU Symp. 171, 175 (1996) [Astrophys. J. 447, L25 (1995)] astro-ph/9504041.

[15] P. Gondolo and J. Silk, Phys. Rev. Lett. 83, 1719 (1999) astro-ph/9906391.

[16] S. D. Hunger et al., Astrophys. J. 481 (1997) 205.

[17] P. Sreekumar et al. [EGRET Collaboration], Astrophys. J. 494, 523 (1998) astro-ph/9709257.

[18] A. W. Strong, I. V. Moskalenko and O. Reimer, Astrophys. J. 613, 962 (2004) astro-ph/0406254.

[19] T. C. Weekes, Very High-Energy Gamma-Ray Astronomy, IOP Publishing LTD, Philadelphia, 2003.

[20] L. Bergstrom, P. Ullio and J. H. Buckley, Astropart. Phys. 9, 137 (1998) astro-ph/9712318.

[21] G. Bertone, D. Hooper and J. Silk, Phys. Rept. 405, 279 (2005) hep-ph/0404175. 